\title{
Strain-engineered graphene through a nanostructured substrate. I. Deformations
}

\author{
M. Neek-Amal ${ }^{1,2}$ and F. M. Peeters ${ }^{2}$ \\ ${ }^{1}$ Department of Physics, Shahid Rajaee Teacher Training University, Lavizan, Tehran 16785-136, Iran \\ ${ }^{2}$ Departement Fysica, Universiteit Antwerpen, Groenenborgerlaan 171, B-2020 Antwerpen, Belgium
}

(Received 6 February 2012; published 22 May 2012)

\begin{abstract}
Using atomistic simulations we investigate the morphological properties of graphene deposited on top of a nanostructured substrate. Sinusoidally corrugated surfaces, steps, elongated trenches, one-dimensional and cubic barriers, spherical bubbles, Gaussian bumps, and Gaussian depressions are considered as support structures for graphene. The graphene-substrate interaction is governed by van der Waals forces and the profile of the graphene layer is determined by minimizing the energy using molecular dynamics simulations. Based on the obtained optimum configurations, we found that (i) for graphene placed over sinusoidally corrugated substrates with corrugation wavelengths longer than $2 \mathrm{~nm}$, the graphene sheet follows the substrate pattern while for supported graphene it is always suspended across the peaks of the substrate, (ii) the conformation of graphene to the substrate topography is enhanced when increasing the energy parameter in the van der Waals model, (iii) the adhesion of graphene into the trenches depends on the width of the trench and on the graphene's orientation, i.e., in contrast to a small-width $(3 \mathrm{~nm})$ nanoribbon with armchair edges, the one with zigzag edges follows the substrate profile, (iv) atomic-scale graphene follows a Gaussian bump substrate but not the substrate with a Gaussian depression, and (v) the adhesion energy due to van der Waals interaction varies in the range $[0.1-0.4] \mathrm{J} / \mathrm{m}^{2}$.
\end{abstract}

DOI: 10.1103/PhysRevB.85.195445

PACS number(s): 65.40.De

\section{INTRODUCTION}

Geometrically structured substrates affect various properties of graphene $e^{1,2}$ and can prevent the crumpling of graphene which is typical for freestanding graphene without a support. ${ }^{3}$ Before graphene's discovery in 2004, the study of $2 \mathrm{D}$ membranes over corrugated substrates was an important branch of soft-condensed matter physics with, e.g., applications in biological systems. ${ }^{4,5}$ Recently, particular attention was focused on the various properties of graphene on top of a substrate. Substrates can induce corrugations, modify the electric conductance, and deform graphene. ${ }^{6,7}$ The electrostatic interaction of graphene on a substrate can be understood as due to the van der Waals (vdW) interaction of graphene with the metallic gate below the substrate, the electrostatic forces between graphene and the polarized substrate, the water between the substrate and graphene, and impurities between graphene and the substrate. ${ }^{8}$ The vdW interaction includes attractive and repulsive terms, which are widely used and extensively investigated in soft matter. ${ }^{9}$ The usual dispersion interaction for the attractive part of the $\mathrm{vdW}$ interaction (sum of contributions proportional to $D^{-6}$ ) must be modified for the two $\pi$-conjugated systems at distance $D$, e.g., graphite. ${ }^{10}$ For the vdW interaction between carbon nanostructures and $\mathrm{Si}-\mathrm{C} / \mathrm{SiO}_{2}$ substrates the Lennard-Jones potential (LJ) is widely used and produced both qualitative and quantitative acceptable results. ${ }^{11-16} A b$ initio calculations obtained vdW energy curves between carbon nanostructures (those curves are similar to the LJ function); e.g., the vdW interaction between hexagonal boron nitride sheets, ${ }^{17}$ methane adsorption on graphene, and gas molecule adsorption in carbon nanotubes were found to be LJ-like functions when using $\mathrm{vdW}$-corrected density functional theory. ${ }^{18,19}$

Recently, experimental measurements on the adhesion energy of pressurized monolayer/multilayer graphene on top of a $\mathrm{SiO}_{2}$ substrate showed that the adhesion energy is ultrastrong $\left(\chi \sim 0.3-0.45 \mathrm{~J} / \mathrm{m}^{2}\right)$ which is many times larger than the one reported for typical micromechanical structures and is of the order of solid-liquid adhesion energies..$^{20}$ This adhesion energy is one order of magnitude larger than the upper limit found for water-modified adhesion between graphene and the substrate. Kusminskiy et al. used $\chi=2 \mathrm{meV} \AA^{-2}$ for the pinning of a tethered membrane (as a model for graphene within continuum elasticity theory) and found the possible morphology of graphene over a Gaussian bump and a Gaussian depression. ${ }^{21}$ Their model includes both bending and stretching energies together with a constant pinning energy.

Here, as distinct from previous works, we investigate graphene on top of several nanostructured substrates with different geometrical deformations. We carried out molecular dynamics simulations at $T=300 \mathrm{~K}$ to minimize the energy and find the optimum profile of the deposited graphene membrane. Sinusoidal substrates with different wavelengths, elongated trenches, barriers, bubbles, Gaussian bumps, and Gaussian depressions are considered as geometrical distinct examples of nanostructured substrates. We find that in the case of a sinusoidal substrate with a short wavelength and small energy parameter in the $\mathrm{vdW}$ model (i.e., $\epsilon$ ), graphene does not follow the substrate. For graphene on top of the trench, it is found that zigzag graphene falls into the well but armchair graphene is suspended across the trench. The stress distribution shows that the atoms within the deformed parts are highly stressed. For the boundary conditions of the examined graphene flakes we considered both free and supported (i.e., fixed) in-plane boundaries. We found a significant difference in the obtained graphene profile when on top of a Gaussian bump or at a Gaussian depression; i.e., the graphene sheet over a depression with $1 \mathrm{~nm}$ variance and $1 \mathrm{~nm}$ height does not fall into the depression while it follows a Gaussian bump with the same size. For a Gaussian bump/depression with larger variance, graphene follows both substrates. The square barrier (a cube with $1 \mathrm{~nm}$ side) influences graphene such that an unexpected pyramidal shape is found which surrounds the 
barrier. We studied the vdW energy stored between graphene and the nanoscale Gaussian bump by employing a continuum model for both systems and calculated the variation of the $\mathrm{vdW}$ energy per area as a function of the energy parameter of the model. We also compared our molecular dynamics results for the Gaussian bump/depression to those predicted by the continuum model and found agreement only in case of a large Gaussian bump with weak interaction, i.e., small $\epsilon$.

This paper is organized as follows. In Sec. II the details of the atomistic model are presented. In Sec. III we present the continuum model for the vdW interaction of graphene and various substrates. In Sec. IV we present results for various nanostructured substrates and different boundary conditions. The results are summarized in Sec. V.

\section{ATOMISTIC MODEL}

Classical atomistic molecular dynamics simulation (MD) is employed to simulate large flakes of graphene (GE). The second generation of Brenner's bond-order potential is employed which is able to describe covalent $s p^{3}$ bond breaking and the formation of associated changes in the atomic hybridization within a classical potential. ${ }^{22}$ The Brenner potential terms were taken as

$$
E_{P}=\sum_{i} \sum_{j>i}\left[V^{R}\left(r_{i j}\right)-B_{i j} V^{A}\left(r_{i j}\right)\right],
$$

where $E_{P}$ is the average binding energy, and $V^{R}$ and $V^{A}$ are the repulsive and attractive terms, respectively, where $r_{i j}$ is the distance between the atoms $i$ and $j$ (all relevant parameters in this study are listed in Table I). $B_{i j}$ is called the bond-order factor which includes all many-body effects. $B_{i j}$ depends on the local environment of the bond, i.e., the bond and torsional angles, the bond lengths, and the atomic coordination in the vicinity of the bond. This feature allows the Brenner potential to predict correctly the configurations, the hybridization, and the energies for many different hydrocarbon structures.

The carbon-carbon bond length, $a_{0}$, is $1.42 \AA$. In our model, the origin of the $x y z$ Cartesian coordinate system is set at $(0$, $0,0)$. Here, the two primitive vectors for the GE sublattices, $\mathbf{a}_{1}=\sqrt{3} a_{0} \hat{i}$ and $\mathbf{a}_{2}=\sqrt{3} / 2 a_{0} \hat{i}+3 / 2 a_{0} \hat{j}$, are the two basic vectors of the GE lattice.

In order to model the van der Waals (vdW) interaction between GE and different substrates, we employed the Lennard-Jones (LJ) potential. The LJ potential describes both the repulsive and attractive parts of the vdW energy between two atoms which are nonbonded. The LJ potential is a widely used potential in various simulations. ${ }^{11-16}$ For two interacting uncharged particles, we have

$$
u(r)=\epsilon\left[\alpha(\sigma / r)^{3 m}-\beta(\sigma / r)^{6}\right],
$$

where $r$ is the distance between the two particles, and $\epsilon$ and $\sigma$ are the "energy parameter" and the "length parameter," respectively. Setting the integer numbers to $\alpha=\beta=m=4$ Eq. (2) gives the 12-6 LJ potential function and for $\alpha=$ $2, \beta=m=3$ Eq. (2) gives the 9-6 LJ potential function. ${ }^{23}$ The minimum of $u(r)$ is $r_{\min }=\sigma\left(\frac{m \alpha}{2 \beta}\right)^{1 /(3 m-6)}$ which yields $2^{1 / 6} \sigma$ and $\sigma$ for the $12-6 \mathrm{LJ}$ and 9-6 LJ potential, respectively. Therefore, the equilibrium distance is shorter for the 9-6 LJ potential while the minimum of $u(r)\left(\left.u\right|_{r_{\min }}=-\epsilon\right)$ is the same for both cases. Notice that for the 12-6 LJ potential both attractive and repulsive terms have the same weights in $u(r)$, i.e., $\alpha=\beta$. We will use mostly the $12-6 \mathrm{LJ}$ potential (in some exceptional cases we use the 9-6 LJ potential, which will be mentioned explicitly).

To model the interaction between two different types of atoms such as the carbon atom (C) and a substrate atom (S), we adjust the LJ parameters using the equations $\epsilon_{T}=\sqrt{\epsilon_{C} \epsilon}$ and $\sigma_{T}=\left(\sigma_{C}+\sigma\right) / 2$. For carbon we use the parameters

\begin{tabular}{|c|c|}
\hline$l_{x}, l_{y}$ & The length and width of the simulated graphene membrane \\
\hline$\epsilon, \sigma$ & The energy and length parameters in the van der Waals (vdW) potential for the substrate atoms, Eq. (2) \\
\hline$\alpha, \beta, m$ & Integer numbers defining the power law of repulsive and attractive parts of the vdW potential in Eq. (2) \\
\hline$\eta^{i}, \mathbf{F}^{i}$ & The stress and the force on atom $i$ \\
\hline$f, E_{v d W}$ & Total free energy [Eq. (6)] and vdW energy [Eqs. (3) and (10)] stored in graphene when on top of a substrate \\
\hline$m, \Omega, \mathbf{v}_{i}, \mathbf{r}_{i}$ & Mass, volume, velocity, and position of atom $i$ \\
\hline$N, M$ & Total number of atoms respectively of the simulated graphene and the substrate \\
\hline$\tau, \kappa$ & Surface tension, bending rigidity of the graphene membrane \\
\hline$\chi, T$ & The vdW energy per planar area $A$ and temperature \\
\hline$h_{G}(x, y), h_{S}(x, y)$ & Height of the graphene membrane and the substrate at $(x, y)$ \\
\hline$E_{P}, B_{i j}, V_{R}, V_{A}$ & Total bond energy of graphene, bond order, repulsive and attractive terms in the Brenner potential, Eq. (1) \\
\hline$v, \Sigma_{S}, \Sigma_{G}$ & Hamaker constant, the density of the simulated substrate and graphene membrane, respectively \\
\hline$\lambda, \theta(x)$ & The wavelength and the step function \\
\hline$R, g(\mathbf{r})$ & The radius of Gaussian bump and the determinant of the metric tensor \\
\hline$h_{0}$ & The amplitude of the sinusoidal waves or height (depth) of Gaussian bump/bubble/barrier (depression or trench) \\
\hline$h_{1}, d$ & A shift or vertical distance between graphene and the substrate and the width of the trenches/barriers \\
\hline$u(r), r_{\min }$ & The Lennard-Jones potential energy and its minimum distance \\
\hline$a_{0}, \mathbf{a}_{1}, \mathbf{a}_{2}$ & Carbon-carbon bond length and two basic vectors of the graphene lattice \\
\hline$\ell$ & Lattice parameter of the substrate lattice \\
\hline$r_{c}, r_{0}$ & Cutoff distance in vdW interaction, upper limit of the integrals in Eq. (10) \\
\hline$r_{i j}$ & Distance between a lattice site of graphene $(i)$ and a lattice site of the substrate $(j)$ \\
\hline$\Delta r_{12}$ & Distance between a surface element of the graphene membrane and a surface element of the substrate \\
\hline
\end{tabular}

TABLE I. List of all relevant parameters used in the paper. 
$\sigma_{C}=3.369 \AA$, and $\epsilon_{C}=2.63 \mathrm{meV}$. For the substrate atoms we vary $\sigma$ in the range $(2.5 \AA, 3.5 \AA)$ and $\epsilon$ in the range (10.0 meV, $140.0 \mathrm{meV}$ ), where the lower limits are typically for insulators, e.g., $\mathrm{SiO}_{2},{ }^{11}$ and the upper limits are typical for metallic substrates, e.g., $\mathrm{Na}, \mathrm{K}$, etc. ${ }^{24,25}$ Notice that the main difference between the two sets of parameters is the energy parameter $(\epsilon)$ which is varied over more than one order of magnitude.

The total vdW energy stored between GE with $N$ atoms and a substrate with $M$ atoms can be written as

$$
E_{v d W}^{A}=\sum_{i=1}^{N} \sum_{j=1}^{M} u\left(r_{i j}\right)
$$

where $r_{i j}=\left|\mathbf{r}_{i}-\mathbf{r}_{j}\right|$ and $\mathbf{r}_{i}$ refers to the position of the $i$ th carbon atom of $\mathrm{GE}$ and $\mathbf{r}_{j}$ refers to the $j$ th atom of the substrate. Often in MD simulations, one approximates the above sums by including only the nearest- atoms in order to reduce the number of interactions. Such an approach is accurate in the case of short-range potentials. Regarding the cutoff distance appropriate for the LJ potential $r_{c}=3 \sigma$, only those substrate atoms inside a sphere having radius $r_{c}$ with origin at the position of the $i$ th atom of GE interact most strongly with the $i$ th atom, while outside this sphere, the interaction strength decreases very fast. Therefore, in practice for each $i$, the sum over $M$ can be truncated and limited to the atoms inside a sphere with radius $r_{c}$. This is done by employing a neighbor list in our MD simulation. In our study, the number of GE atoms is $N=14400$ (which is equivalent to a graphene sheet with dimension $l_{x}=19.17 \mathrm{~nm}$ and $\left.l_{y}=19.67 \mathrm{~nm}\right)$ and the number of substrate atoms is $M=6000$ (only in the case of the Gaussian bump we performed a simulation with $N=72000$ and $M=35000$ ).

The adhesion energy can be obtained using $a b$ initio calculations and can be estimated using classical models (e.g., LJ potential). The present-day patterns of deformation of large-scale GE on top of substrates is beyond reach of traditional $a b$ initio methods. Our classical vdW model as based on the LJ potential are able to simulate the realistic sizes and gives a vdW energy (the main term in the binding energy) between two nonbonded systems. Note that several $a b$ initio calculations have demonstrated that the $\mathrm{vdW}$ interaction between nanoscale objects can be well approximated by a LJ potential. ${ }^{17-19}$

The gradient of the total potential energy, i.e., $E_{\text {total }}=$ $E_{P}+E_{\text {bind }}^{A}$, is the force experienced by the $i$ th carbon atom, $\mathbf{F}_{i}=-\nabla_{i} E_{\text {total }}$. In common molecular dynamics simulations Newton's second law should be solved numerically in order to determine the path of motion of the atoms. In this study, the equations of motion were integrated using a velocity-Verlet algorithm with a time step of $0.5 \mathrm{fs}$ and the temperature was held constant at $T=10 \mathrm{~K}$ by a Nosé-Hoover thermostat.

The atomic stress experienced by each $i$ th atom can be expressed as ${ }^{26,27}$

$$
\eta_{\mu \nu}^{i}=\frac{1}{\Omega}\left(\frac{1}{2} m v_{\mu}^{i} v_{\nu}^{i}+\sum_{j \neq i} r_{i j}^{v} F_{i j}^{\mu}\right)
$$

where the inner summation is over all the carbon atoms which are neighbors of the $i$ th atom which occupies a volume
$\Omega=4 \pi a_{0}^{3} / 3$. The quantities $m$ and $v^{i}$ denote the mass and velocity of the $i$ th atom and the scaler $r_{i j}^{v}$ is the $v$ component of the distance between atoms $i$ and $j . F_{i j}^{\mu}$ is the force on the $i$ th atom due to the $j$ th atom in the $\mu$ direction. We have used this expression to calculate the stress on each atom. In order to be able to visualize the stress distribution on the GE atoms, we colored the atoms according to the value of the dimensionless stresses; i.e., green (red) is related to the minimum (maximum) possible stress.

\section{CONTINUUM APPROACH}

Evaluation of the vdW contribution of the stored energy in the deformed GE with average density $\Sigma_{G}$, due to the interaction with a substrate with average density $\Sigma_{S}$, is obtained after the integration of the vdW potential over both GE and the substrate surfaces. Here, we present for comparative purposes a continuum model for the stored vdW energy between the GE membrane and the substrate. Such an approach can be used to calculate the vdW energy stored between two objects. ${ }^{4,16,28}$

In the absence of external pressure, the total free energy of a membrane consists of three terms, i.e., bending, stretching, and vdW terms, which are given by

$$
f=\iint d x d y\left[\tau\left(\nabla h_{G}(x, y)\right)^{2}+\kappa\left(\nabla^{2} h_{G}(x, y)\right)^{2}\right]+E_{v d W}^{C},
$$

where $\tau$ and $\kappa$ are the surface tension and the bending rigidity of the membrane, respectively. The two first terms in Eq. (5) are relevant to the bending and stretching energies of $\mathrm{GE}^{21}$ and the third term, $E_{v d W}^{C}$, is the total vdW contribution of the interaction between the substrate and the membrane. $E_{v d W}^{C}$ includes two repulsive and attractive terms. The stored adhesion energy per area is determined by ${ }^{4}$

$$
\chi_{T}=\left(\frac{f_{\min }}{A}-\tau\right),
$$

where $f_{\min }$ is the minimum of the total free energy when the membrane takes its optimum configuration. $A$ is the projected area onto the $x-y$ plane; i.e., $A=\int d x d y$. Equation (6) was used by Swain et al. ${ }^{4,5}$ to estimate the adhesion energy of typical soft membranes over different substrates. In Ref. 21 the adhesion part in the free energy was taken as a coupling constant. Assuming a planar local relative height coordinate function for the vdW interaction energy between the substrate and the soft membrane, i.e., the Deryagin approximation, the $\mathrm{vdW}$ energy is approximated by

$$
E_{v d W}^{C}=\iint d x d y\left[V_{0}+\psi\left(\delta h^{2}\right)\right],
$$

where $\psi$ is a function of the height increment $\delta h=h_{G}(x, y)-$ $h_{S}(x, y)$ and $V_{0}$ is a constant. Substituting Eq. (7) in Eq. (5) and minimizing the total free energy with respect to $h_{G}(x, y)$ results in the following differential equation: ${ }^{4,5}$

$$
\left(\kappa \nabla^{4}-\tau \nabla^{2}+v\right) h_{G}(x, y)=v h_{S}(x, y),
$$

where $v$ is proportional to the Hamaker constant $(\propto$ $\left.\epsilon \sigma^{6} \Sigma_{S} \Sigma_{G}\right)$. Equation (8) can be solved in Fourier 
space: $:^{4,5}$

$$
h_{G}(\mathbf{k})=\frac{h_{S}(\mathbf{k})}{1+k^{2} \xi_{\tau}+k^{4} \xi_{\kappa}},
$$

where $k$ is the wave vector, $\xi_{\tau}=\tau / v$, and $\xi_{\kappa}=\kappa / v$. Equation (9) was used to find the optimum configuration of a soft membrane on top of corrugated substrates. ${ }^{4,5}$

Here we assume that the vdW energy is not localized and use the LJ potential for the interaction between graphene and the substrate. This gives us the vdW contribution to the adhesion energy, i.e., $\chi$. We assume that both substrate and membrane are homogenous and continuous materials. The obvious difference between the atomistic model and the continuum model is the absence of the chirality effect. The LJ potential energy between the GE membrane and the continuum substrate is given by

$$
E_{v d W}^{C}=\iint_{G, S} \zeta\left(\mathbf{r}_{1}\right) \zeta\left(\mathbf{r}_{2}\right) u\left(\Delta r_{12}\right) d s_{1} d s_{2},
$$

where $\quad \Delta r_{12}=\left|\mathbf{r}_{1}-\mathbf{r}_{2}\right|$ and $\zeta\left(\mathbf{r}_{1}\right)=\Sigma_{G}\left(\mathbf{r}_{1}\right) g_{G}\left(\mathbf{r}_{1}\right)$ and $\zeta\left(\mathbf{r}_{2}\right)=\Sigma_{S}\left(\mathbf{r}_{2}\right) g_{S}\left(\mathbf{r}_{2}\right)$. Here, $\Sigma_{G}=2 /\left|\mathbf{a}_{1} \times \mathbf{a}_{2}\right|$ and $\Sigma_{S}$ are the mean surface density of carbon atoms in the GE and the substrate lattice, respectively. The substrate density is $\Sigma_{S}=\ell^{-2}$ which is equivalent to a (100) surface of a crystal with lattice parameter equal to $\ell$. The scaler $g\left(\mathbf{r}_{i}\right)=\{1+$ $\left.\left[\vec{\nabla} h\left(x_{i}, y_{i}\right)\right]^{2}\right\}^{1 / 2}$ is defined by the appropriate transformation of a surface element in curvilinear coordinates into a two-dimensional planar surface $(x-y$ plane in Cartesian coordinates), i.e., the determinant of the metric tensor. In Eq. (10) the position vector $\mathbf{r}_{i}=\left(x_{i}, y_{i}, h\left(x_{i}, y_{i}\right)\right)$ refers to the position of the surface element $d s_{i}$ of the GE membrane $(i=1)$ or substrate $(i=2)$. As already mentioned, because of the short-range nature of the $\mathrm{LJ}$ potential, we assume that the main contribution of the vdW energy is due to the interaction with the outer surface of the substrate. This model gives a good insight into the vdW adhesion energy between GE and various substrates. For the continuum model we will give only the results for GEs on top of a Gaussian bump/depression. Notice that it is analytically impossible to minimize Eq. (5) by substituting $E_{v d W}^{C}$ [Eq. (10)]. Therefore, we used the optimum configuration for the GE membrane as obtained from our MD simulations.

\section{RESULTS AND DISCUSSION}

In this study we investigate several different geometries for the substrate which can be realized experimentally. The substrate atoms are assumed to be rigid during the simulations which is a reasonable approximation due to the different atomic-vibrations time scale in graphene and the substrate. In order to model the substrate, a (100) surface having lattice parameter $\ell=3 \AA$ is used which is a typical lattice parameter. The density of sites in the substrate is $\Sigma_{S}=\ell^{-2}$. Since the interaction between the substrate and graphene is weak and of short range (i.e., van der Waals interaction) the main contribution to the mutual stored energy and to the force between graphene and the substrate comes from the upper layer of the substrate. We can show this explicitly by calculating the energy and force as function of the number of atomic layers in the substrate for one of our samples [we took the case of graphene over a Gaussian bump; see Fig. 12(a)]. The total energy can be written as

$$
E_{v d W}^{A}(L)=\sum_{n=1}^{L} \sum_{i, j}^{N, M} u_{n}\left(r_{i j}\right),
$$

where $u_{n}$ is the contribution of the $n$th layer and $L$ is the number of considered substrate layers. For instance, for graphene on top of a Gaussian bump the attractive and repulsive parts are proportional to $\left[\rho^{2}+(z+n \ell)^{2}\right]^{-3}$ and $\left[\rho^{2}+(z+n \ell)^{2}\right]^{-6}$, respectively [here $\rho(z)$ is the planar (vertical) distance between an atom in graphene and one in the top layer of the substrate], which decrease fast with $n$. Recalling the discussion below Eq. (3) we found that [Eq. (11)] for the considered system around the central points $(r<30 \AA)$ the top layer $(n=1)$ contributes almost $99 \%$ of the total energy, the second layer contributes $1 \%$, and the contribution of the other layers is negligible. This motivated us to restrict our study to the top substrate layer which helps us considerably to minimize the CPU time. Note that for larger $\Sigma_{S}$ and smaller $\sigma_{T}$ the contribution of the second layer will increase.

The heights of the graphene and the substrate atoms at each point $(x, y)$ are denoted by $h_{G}(x, y)$ and $h_{S}(x, y)$, respectively. The calculations are done for two different boundary conditions: (i) free boundary condition and (ii) supported boundary conditions which prevents in-plane movements for two longitudinal ends of GE. The two atom rows at the longitudinal ends were taken in the zigzag direction (in most cases) and they are allowed to move in the $z$ direction.

\section{A. Sinusoidal substrates: Free boundary condition}

A sinusoidal deformation of the substrate along the $x$ direction is given by

$$
h_{S}(x, y)=h_{0} \sin (k x),
$$

where $k=2 \pi / \lambda$ and the amplitude is $h_{0}$. We used different wavelengths, i.e., $\lambda=2,3$, and $4 \mathrm{~nm}$, and two sets of $\sigma$ and $\epsilon$, i.e., $(3.5 \AA, 10.0 \mathrm{meV})$ and $(3.4 \AA, 100.0 \mathrm{meV})$, with fixed $h_{0}=$ $0.5 \mathrm{~nm}$. At the start of the simulation, we put a flat graphene sheet on top of this substrate at $h_{G}(x, y)=h_{S}(x, y)+r_{\min }$. We choose the $x$ direction to be the armchair direction.

After $0.5 \mathrm{~ns}$ of the MD simulation, GE found its optimum configuration which is deformed and corrugated. Figure 1 shows six snapshots of free GE (upper corrugated sheets in each panel) over three different substrates (filled circles in each snap shot). In Figs. 1(a)-1(c) the vdW parameters were set to $\sigma=3.5 \AA, \epsilon=10.0 \mathrm{meV}$ and in Figs. 1(d) $-1(\mathrm{f})$ the $\mathrm{vdW}$ parameters were set to $\sigma=3.4 \AA, \epsilon=100.0 \mathrm{meV}$. The wavelength in Figs. 1(a) and 1(d) is $2 \mathrm{~nm}$. Notice that for $\lambda=2 \mathrm{~nm}$ GE does not follow the substrate, i.e., $h_{G}(x, y) \neq h_{1}+h_{S}(x, y)$, where $h_{1}$ is a vertical shift of the order of graphite's layer distance, i.e., 3.4 A. By increasing the wavelength or $\epsilon$, GE follows more closely the substrate profile, i.e., $h_{G}(x, y) \cong h_{1}+h_{S}(x, y)$. Increasing $\epsilon$ yields stronger adhesion and deforms GE [Fig. 2 shows zoomed versions of Fig. 1(c)]. Therefore, according to our MD simulations, the shortest wavelength which makes GE's profile similar to the substrate's profile is larger than $\lambda=2 \mathrm{~nm}$. In all figures the colors indicate the stress distribution. Notice that at the 


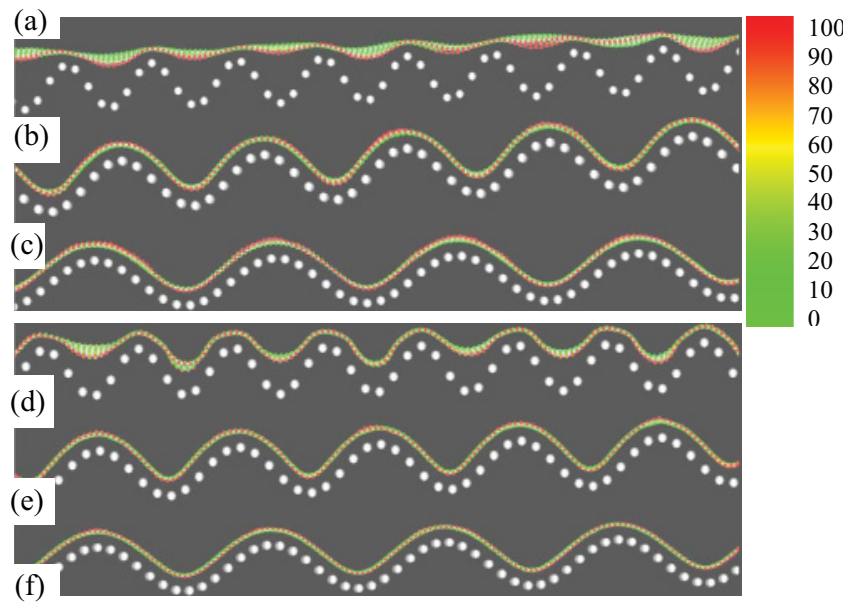

FIG. 1. (Color online) Side views of the optimum configuration of graphene on top of sinusoidal substrates with different wavelengths. The filled white circles are the substrate atoms. The parameters in (a), (b), and (c) are $\sigma=3.5 \AA, \epsilon=10.0 \mathrm{meV}$ and in (d), (e), and (f) are $\sigma=3.4 \AA$ and $\epsilon=100.0 \mathrm{meV}$. The wavelengths are $\lambda=2 \mathrm{~nm} \mathrm{[(a),}$ (d)], $\lambda=3 \mathrm{~nm}[(\mathrm{~b}),(\mathrm{e})]$, and $\lambda=4 \mathrm{~nm}[(\mathrm{c})$, (f)]. For the graphene sheet, the colors represent the stress distribution; the highest stress is denoted by red and the lowest by green.

boundaries we always have red colors (i.e., maximum stress) because of the presence of dangling bonds. In the other parts, the distribution of stress is uniform (green colors). Notice that in each particular system we scaled the colors by the highest stress.

Here we compare our results to those predicted by continuum elasticity theory for a membrane on top of sinusoidal surfaces. The possible solution of Eq. $(8)^{4}$ for a membrane on top of a deformation given by Eq. (12) is

$$
h_{G}(x, y)=\frac{h_{0} \sin (k x)}{1+k^{2} \xi_{\tau}+k^{4} \xi_{\kappa}} .
$$

For longer wavelengths, or small $\kappa$ and $\tau$, this solution gives $h_{G}(x, y) \approx h_{S}(x, y)$ which is in agreement with our $\mathrm{MD}$ results for long wavelengths and large $\epsilon$. For graphene membrane $\kappa \sim 1.1 \mathrm{eV}$ and $\tau \sim 10 \mathrm{eV} / \AA^{2}$ (taken of the order

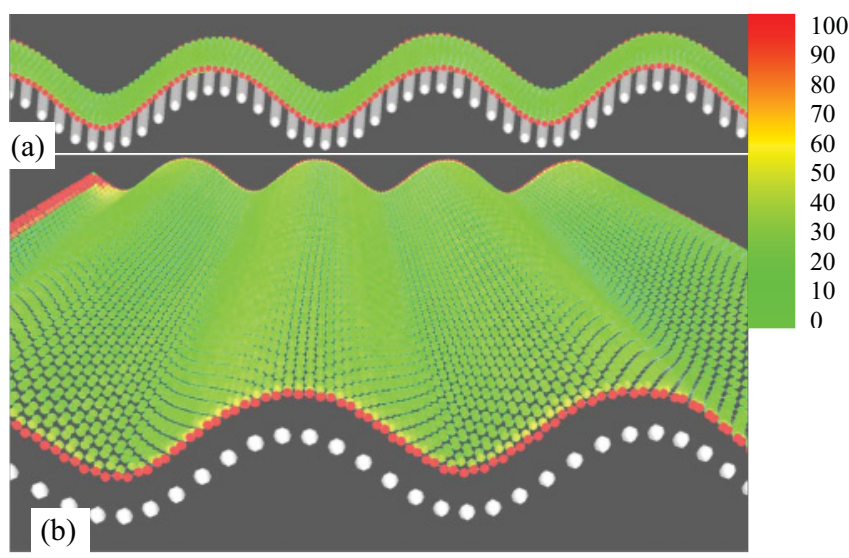

FIG. 2. (Color online) Two side views of Fig. 1(c). For the graphene sheet, the colors indicate the stress distribution. The substrate atoms are indicated by filled white circles below graphene. The sinusoidal substrate is shown only by the first front row of atoms.

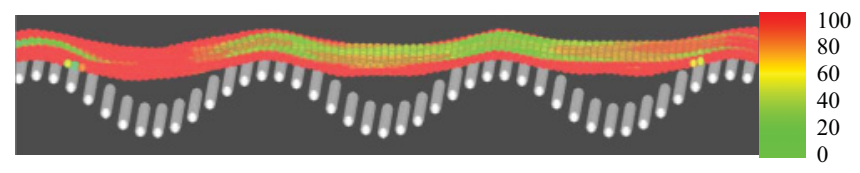

FIG. 3. (Color online) The optimum configuration of graphene over a substrate with wavelength $\lambda=4 \mathrm{~nm}$. Graphene is supported by the longitudinal ends while it can move along the $z$ direction. Here $\epsilon=10 \mathrm{meV}$ and $\sigma=3.4 \AA$ for the $12-6 \mathrm{LJ}$ potential [see also Fig. 1(c) which shows graphene with free boundaries over the same substrate].

of the Lamé coefficients) and Eq. (13) is valid if $v \gg \kappa, \tau$, which are related to both large $\epsilon$ and $\Sigma_{S}$. The stiffer membrane with larger $\kappa$ and $\tau$ can not curve easily and stronger adhesion due to a larger $v(\propto \epsilon$, i.e., stronger adhesion) is required. By using $\sigma=\left(\sigma_{C}+\sigma_{S}\right) / 2=(3.369+3.4) / 2 \AA, \epsilon=$ $\sqrt{2.63 \times 10} \mathrm{meV}, \Sigma_{G}=0.225 \AA^{-2}$, and $\Sigma_{S}=0.026 \AA^{-2}$ yields the Hamaker constant $\approx 4 \pi^{2} \epsilon \sigma^{6} \Sigma_{G} \Sigma_{S}=1.78 \mathrm{eV}$ and consequently $v \sim 0.1$ and thus $k^{2} \xi_{\tau} \ll 1$ and $k^{4} \xi_{\kappa} \ll 1$ yield $\lambda \gg 4.7 \AA$ and $\lambda \gg 5.6 \AA$, respectively, which are in agreement with our MD finding, i.e., $\lambda>20 \AA$.

\section{B. Sinusoidal substrates: Supported boundary condition}

Here, we impose the supported boundary condition on the longitudinal ends (which is mostly taken to be the zigzag direction). In this case the atoms at $x= \pm l_{x}$ are not allowed to move in-plane while they are allowed to move in the $z$ direction. In this section we use the $12-6 \mathrm{LJ}$ potential with parameters $\epsilon=10 \mathrm{meV}$ and $\sigma=3.4 \AA$. We repeated the above simulations by applying the supported boundary condition along the zigzag direction for graphene on top of a substrate with $\lambda=4 \mathrm{~nm}$. Figure 3 shows the optimum configuration of the deposited graphene over this substrate after minimization. Notice that the obtained deformation is different from those for free graphene over the same substrate, Fig. 1(c). The reason is the supported boundary (i.e., fixed in-plane) at the two longitudinal ends. Graphene does not follow the substrate, but the lateral edges (along the $x$ direction at $\pm l_{y} / 2$ ) feel a much large stress as compared to Fig. 1(c). Graphene is suspended across the periodic peaks with a small curvature between them. The larger $\epsilon$ enhances the latter effect. Therefore, the vdW energy is not dominating the bending energy of GE.

\section{Step: Free boundary condition}

The second class of interesting substrate configurations are steps which were recently studied in an experiment to measure the electronic structure and morphology of deposited graphene $^{29}$

$$
h_{S}(x, y)=h_{0} \theta(x),
$$

where $\theta(x)$ is the Heaviside step function with step height of $h_{0}=1 \mathrm{~nm}$ and with $\Sigma_{S}$ density of sites. GEs with both armchair and zigzag directions are put on top of the steps.

Figure 4 shows two snapshots of the optimum configurations [an armchair GE with two different directions of view in (a) and (b) and a zigzag GE with two different directions of view in (c) and (d)] of free GEs on top of steps. All GEs follow the steps except around $x \approx 0$ where GE is bent 

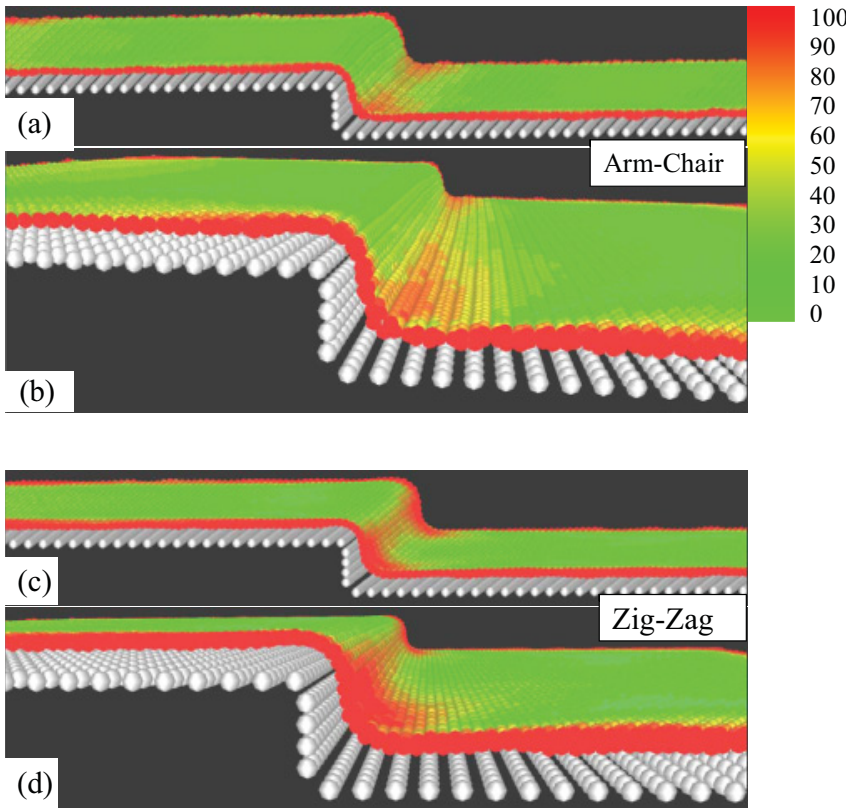

FIG. 4. (Color online) Armchair [(a), (b)] and zigzag [(c), (d)] graphene on top of two steps of height $1 \mathrm{~nm}$ shown along two different angles of view. The highest stressed atoms are shown by red and the lowest by green. The substrate atoms are shown by filled circles below graphene.

in a continuous fashion and does not follow the substrate. There are no considerable differences between the optimum configurations of the free armchair [Figs. 4(a) and 4(b)] and free zigzag [Figs. 4(c) and 4(d)] GEs on top of the step. Indeed, the wall atoms at $x=0$ shift (due to adhesion) in both the left and the right parts of the GE toward $x=0$.

\section{Step: Supported boundary condition}

In Fig. 5 the optimum configuration of GE along the armchair direction with supported boundary condition is shown which is over a sharp step defined by Eq. (14). Notice that there is a significant difference between the deformation obtained here and the one depicted in Fig. 4. The curvature around the step $(x \approx 0)$ is different and all atoms of GE feel more or less equal stress. The wall at $x=0$ adheres to both the left and the right part of the GE but the supported ends prevent full adhesion of GE to the wall, especially for the right-hand side of GE.

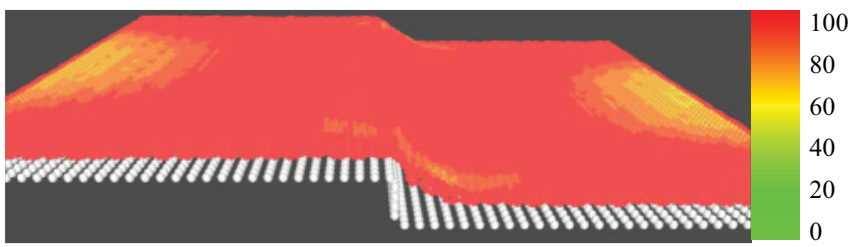

FIG. 5. (Color online) The optimum configuration of armchair graphene over a step located at $x=0$ with supported longitudinal edges [see also Figs. 4(a) and 4(b) which show graphene with free boundaries over the same substrate]. Here $\epsilon=10.0 \mathrm{meV}$ and $\sigma=$ $3.4 \AA$ for the $12-6 \mathrm{LJ}$ potential.
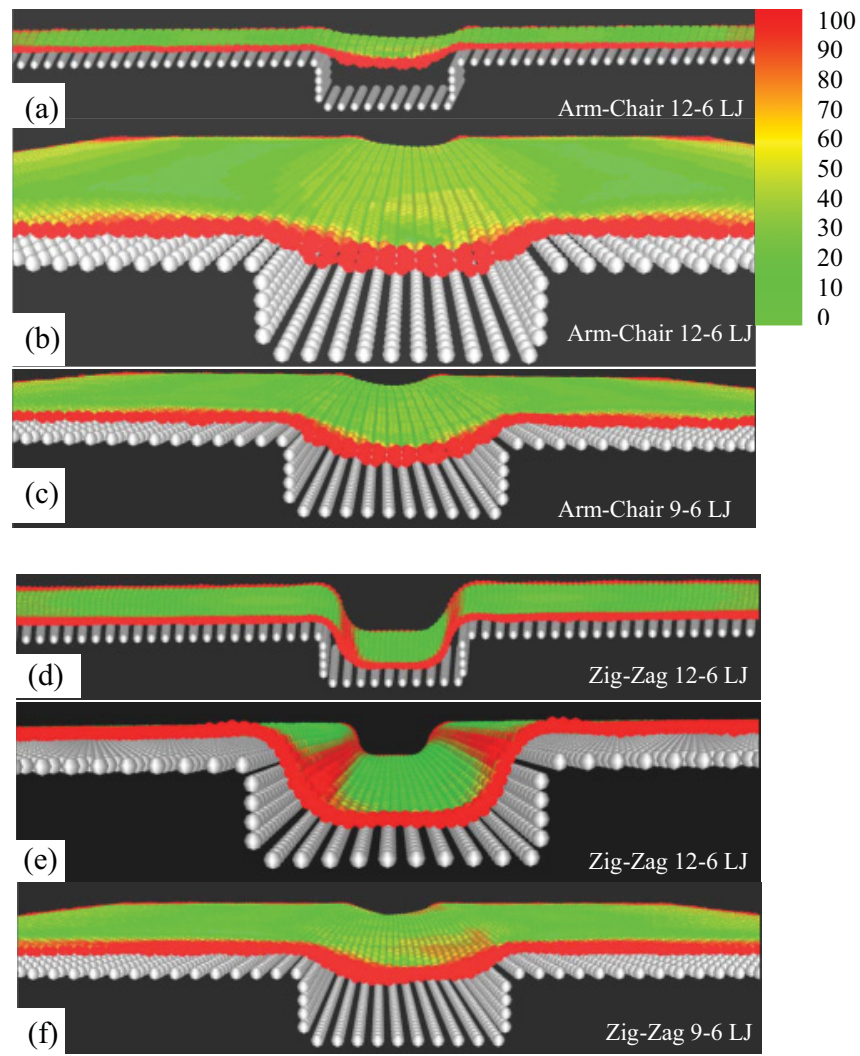

FIG. 6. (Color online) Armchair [(a), (b), (c)] and zigzag [(d), (e), (f)] graphene on top of trenches $1 \mathrm{~nm}$ deep and $3 \mathrm{~nm}$ wide along the $y$ direction. Panels (a) and (b) [and also (d) and (e)] are the same with different angles of view. For a graphene sheet, the colors indicate the stress distribution. The substrate atoms are indicated by filled circles below the GE. Zigzag graphene follows the trench in contrast to armchair graphene. In (a), (b), (d), (e) and in (c), (f) we used the 12-6 LJ and the 9-6 LJ potential, respectively.

\section{E. Trench: Free boundary condition}

The other important substrate that we studied here is an elongated trench

$$
h_{S}(x, y)=h_{0} \theta\left(x^{2}-d^{2}\right),
$$

with two walls located at $x= \pm d= \pm 1.5 \mathrm{~nm}$ with step height of $1 \mathrm{~nm}$ and with $\Sigma_{S}$ density of sites. Figure 6 shows two snapshots of GE on top of such trenches [an armchair GE with two different angles of view in (a), (b), and (c) and a zigzag GE with two different angles of view in (d), (e), and (f)]. After MD minimization zigzag GE follows the trench except around $x \approx \pm 1 \mathrm{~nm}$. In this region, zigzag GE is bent and does not follow the substrate. There is a significant difference between the optimum configurations of armchair [Figs. 6(a) and 6(b)] and zigzag [Figs. 6(d) and 6(e)] GEs. An armchair GE does not follow the trench as well as a zigzag GE. We attribute this effect to the larger number of atoms of zigzag GE (as compared to armchair GE) in the well region $(|x| \leqslant d)$, which yields a strong attractive force on the GE atoms due to the substrate atoms within the well's wall. Recently, Lu et al ${ }^{23}$ studied wider trenches with $2 d=28.6 \mathrm{~nm}$ using a 9-6 LJ potential in order to find the vdW adhesion of GE membranes. In our study we also used the 9-6 LJ potential and found different deformations as 


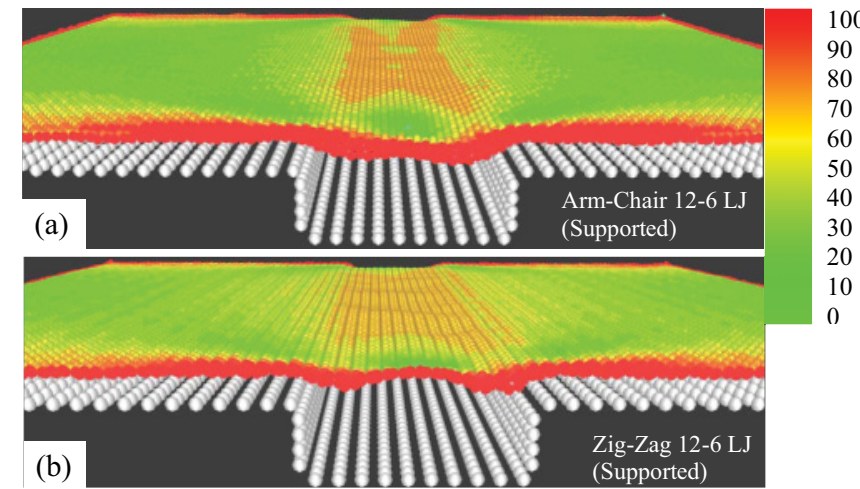

FIG. 7. (Color online) The optimum configuration of armchair (a) and zigzag (b) graphene over two trenches located at $|x|<1.5 \mathrm{~nm}$ where both ends were supported in the $x-y$ plane [see also Figs. 6(a) and $6(d)$ which show two graphene membranes with free boundaries over the same substrate]. Here $\epsilon=10 \mathrm{meV}$ and $\sigma=3.4 \AA$ for $12-6 \mathrm{LJ}$ potential.

compared to the 12-6 LJ potential; see Figs. 6(c) and 6(f). This is due to the different strength of both attractive and repulsive parts in the two models.

\section{F. Trench: Supported boundary conditions}

In Fig. 7 we show the optimum configuration of armchair (a) and zigzag (b) graphene with supported boundary condition on top of the trench defined by Eq. (15). There is a significant difference between the deformation obtained here and those depicted in Fig. 6. The curvature for $|x|<d$ is very different and GE atoms around the well feel a lower stress as compared to the one shown in Fig. 6. Here both armchair and zigzag GEs do not follow the substrate and were suspended over the wells which is a consequence of the supported boundaries. Therefore, by controlling the boundary condition one can clearly control the GE deformation over the substrate.

\section{G. Barriers: Free boundary condition}

A barrier in the middle of the substrate is the inverse situation of the previous ones. An elongated barrier in the $y$ direction is parameterized as

$$
h_{S}(x, y)=h_{0} \theta\left(x^{2}-d^{2}\right),
$$

with two walls at $x= \pm d= \pm 1.5 \mathrm{~nm}$ with step height of $1 \mathrm{~nm}$ and with $\Sigma_{S}$ density of sites. Figures 8(a) and 8(b) show two snapshots of armchair GE (with two different angles of view) on top of the elongated barrier. As we see the stressed regions are located around $x= \pm d$. GE does not follow the rectangular shape of the barrier in this part.

Another interesting case is the one of a substrate that consists of a cubic barrier in the middle [see the inset (i) in Fig. 8(c)]

$$
h_{S}(x, y)=h_{0} \theta\left(x^{2}-d^{2}\right) \theta\left(y^{2}-d^{2}\right),
$$

with four walls at $x, y= \pm d= \pm 1 \mathrm{~nm}$ with step height of $1 \mathrm{~nm}$ and with $\Sigma_{S}$ density of sites. Figures 8(c) and 8(d) show that the optimum configuration is pyramidal shaped [inset (ii) shows this schematically]. This particular deformation is due to the four corners of the cube which strongly repel the
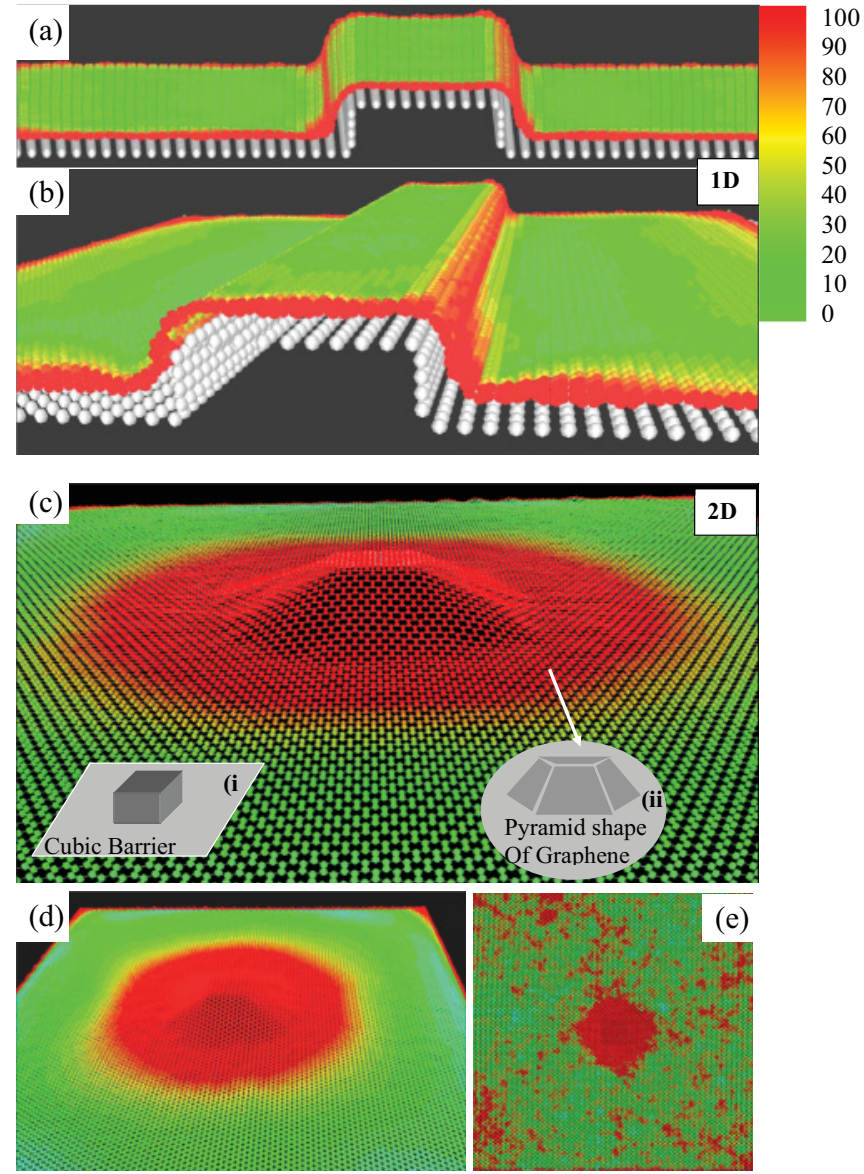

FIG. 8. (Color online) Elongated barrier [(a), (b)] and a cubic barrier [(c), (d)] deformation in the middle of a substrate covered with graphene. The colors indicate the stress distribution in graphene. The inset (i) in (c) shows a schematic model for the cubic barrier and the inset (ii) is a schematic for the optimum configuration of graphene, i.e., a pyramidal shape. Panel (d) is another view of (c). The C-C bond length distribution for (c) [or (d)] is shown in (e), where the red colors are related to the bonds around $1.47 \AA$ and green colors are related to the bonds around $0.140 \AA$. Here $\epsilon=10 \mathrm{meV}$ and $\sigma=3.4 \AA$ for 12-6 LJ potential.

GE. The highest stresses are distributed around the steps (red colors in the $\left|x^{2}+y^{2}\right| \leqslant 3 d$ ). The $\mathrm{C}-\mathrm{C}$ bond lengths in GE are distributed nonuniformly [Fig. 8(e)] but still around the barrier we have a larger stretch in the bond lengths (up to $\simeq 0.147 \mathrm{~nm}$ which are shown by red colors).

\section{H. Barriers: Supported boundary conditions}

Figure 9 shows the optimum configuration of armchair GE in the case of supported boundary condition over two different barriers [defined by Eqs. (16) and (17)]. As we see the stress distribution and the deformations are completely different from those shown for free graphene (see Fig. 8).

\section{Spherical bubble: Free boundary condition}

The next important type of deformation for the substrate that has been realized experimentally ${ }^{30,31}$ is a bubble [see 


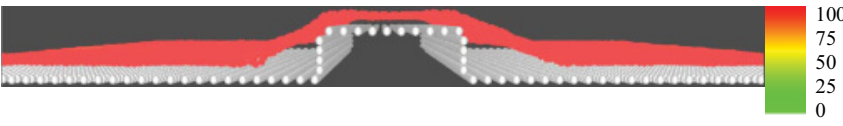

FIG. 9. (Color online) The optimum configuration of armchair graphene over an elongated barrier of size $|x|<1.5 \mathrm{~nm}$ where zigzag edges were supported in the $x-y$ plane (see also Fig. 8 which shows graphene with free boundaries over the same substrate). Here $\epsilon=$ $10 \mathrm{meV}$ and $\sigma=3.4 \AA$ for $12-6 \mathrm{LJ}$ potential.

Fig. 10(b)] which we model by

$$
h_{S}(x, y)=\sqrt{R^{2}-\rho^{2}}+h_{1},
$$

where $R$ is the radius of the bubble and $\rho^{2}=x^{2}+y^{2}$. In order to create a uniform discrete atomistic structure for the bubble, we set $h_{1}=-R / 2$. Figures 10 (a) and 10(c) show the obtained optimum configuration from MD simulation for the GE on top of a bubble with $R=2 \mathrm{~nm}$. The optimum configuration is a Gaussian. In order to produce uniform bubbles, we increased the density of lattice sites in the bubble location where $\Sigma=$ $1 / 4 \AA^{-2}$. Increasing the number of lattice sites on the bubble results in a much larger stressed $\mathrm{GE}$ which influences regions far from the center [see Fig. 10(a)].

\section{J. Spherical bubble on the substrate: Supported boundary condition}

The optimum configuration for the supported graphene over a bubble substrate is shown in Fig. 11. Due to the supported ends GE elongates longitudinally along the supported direction; see inset in Fig. 11.

\section{K. Gaussian bump/depression: Free boundary conditions}

There have been already a few studies that evaluated different properties of a GE membrane in the presence of a
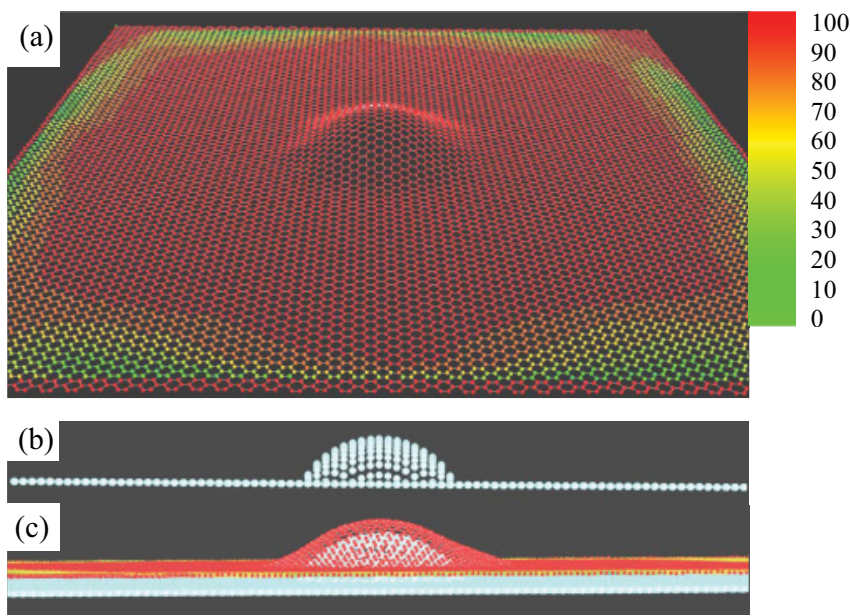

FIG. 10. (Color online) (a) The optimum configuration of the graphene sheet over a bubble with $h_{S}(x, y)=\sqrt{R^{2}-\rho^{2}}-R / 2$ deformation $(R=2 \mathrm{~nm})$. The corresponding substrate is shown in (b). In (c) we show both the GE and the substrate. For a graphene sheet, the colors indicate the stress distribution. The highest stress is shown by red color and the lowest by green. The substrate atoms are indicated by filled white circles below graphene.

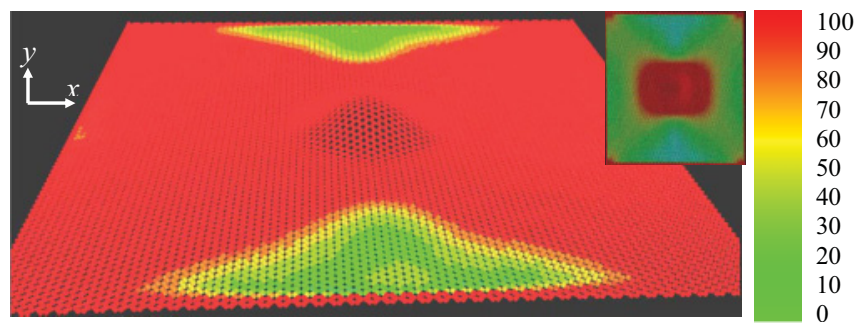

FIG. 11. (Color online) The optimum configuration of armchair graphene with two longitudinal ends supported in the $x-y$ plane on top of a bubble (see also Fig. 10 which shows graphene with free boundaries over the same substrate). The inset shows the elongation of the deformation of graphene along the $x$ direction, i.e., the armchair direction. Here $\epsilon=10 \mathrm{meV}$ and $\sigma=3.4 \AA$ for $12-6 \mathrm{LJ}$ potential.

Gaussian deformation, but those studies did not address the following issues: (i) the creation of the Gaussian deformation in GE using an atomistic-scale deformed substrate; (ii) the question of what are the effects of the vdW energy strength on both the deformation and the adhesion energy at the atomistic scale; (iii) the question of what are the important differences between the deformation due to a bump and due to a depression on the atomistic scale, and (iv) the question of what is the effect of the boundary condition on GE.

The Gaussian bump (protrusion)/depression ${ }^{21,32}$ are parameterized as [Fig. 12(b)]

$$
h_{S}(x, y)= \pm h_{0} \exp \left(-\rho^{2} / 2 \gamma^{2}\right),
$$

where $+h_{0}\left(-h_{0}\right)$ is the height (depth) of the Gaussian bump (depression), $\rho^{2}=x^{2}+y^{2}$, and $\gamma$ is the variance of the Gaussian. Kusminskiy et al. studied recently the pinning of GE over a Gaussian bump in order to find the corresponding attachment/detachment of GE. ${ }^{21}$ Our model is more realistic with relevant length scales for both height and variance of the bumps/depressions. Figure 12(a) shows a snapshot of the optimum configuration of a GE on top of the Gaussian bump

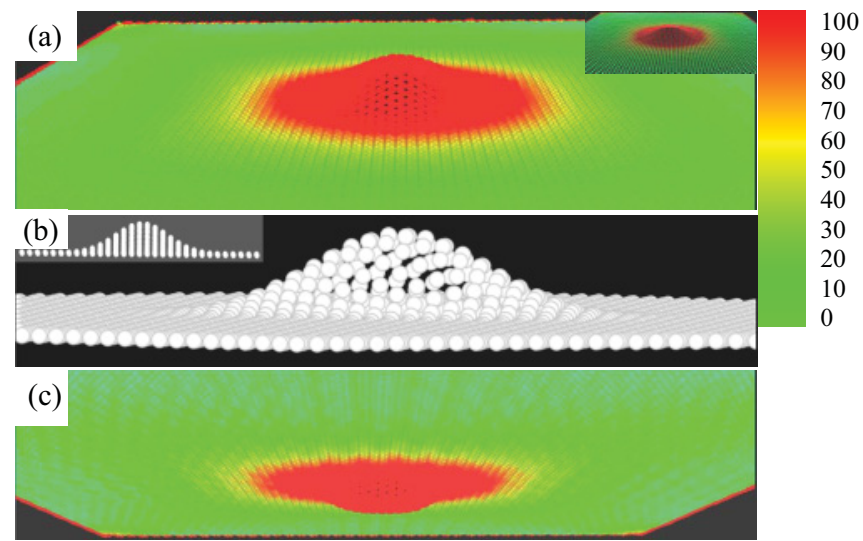

FIG. 12. (Color online) The optimum configurations obtained from molecular dynamics simulations for a graphene sheet places on top of a Gaussian bump (a) Gaussian depression (c) with $h_{S}(x, y)=10 \exp \left(-\rho^{2} / 200\right)(\AA)$. Here we see that graphene on top of the Gaussian bump follows the substrate which is not the case for a depression. For a graphene sheet, the colors indicate the stress distribution. Here $\epsilon=10 \mathrm{meV}$ and $\sigma=3.4 \AA$. Panel (b) is the bumped substrate; i.e., $h_{S}(x, y)=10 \exp \left(-\rho^{2} / 200\right)(\AA)$. 

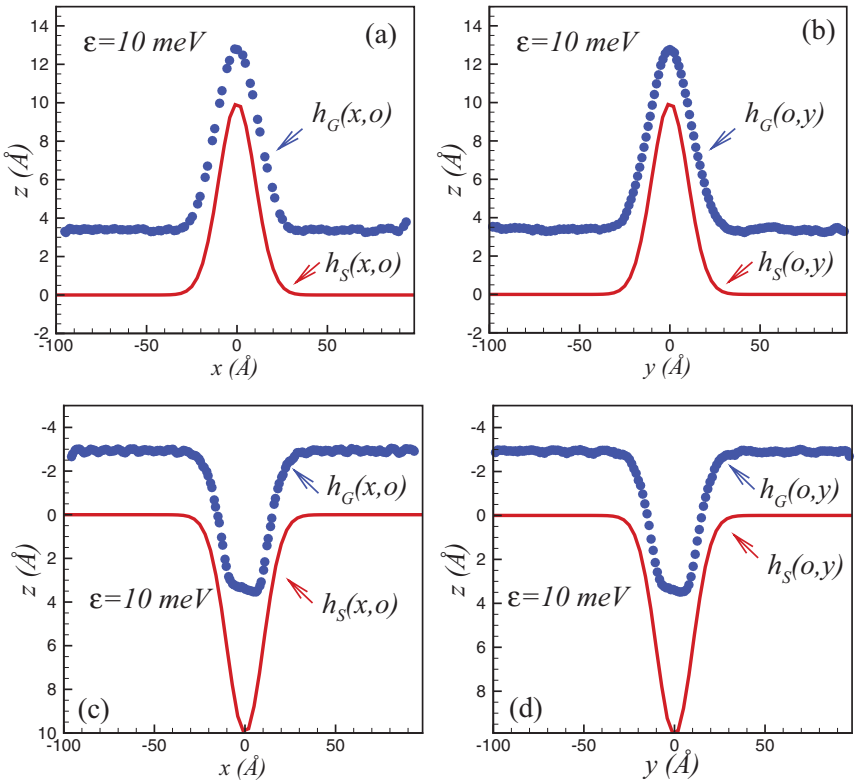

FIG. 13. (Color online) The heights of the substrate (red) and graphene (blue) along $x=0$ and $y=0$ on top of a Gaussian bump/Gaussian depression in the $x$ direction (a)/(c) and $y$ direction (b)/(d) (they are taken from the central portion of those deformations shown in Fig. 12). Circular points indicate the $C$ atoms of graphene and the solid curves are the substrate. Notice that graphene follows the bump which is not the case for a depression. The colors indicate the stress distribution in the graphene sheet. Here $\epsilon=10.0 \mathrm{meV}$ and $\sigma=3.4 \AA$.

[see Fig. 12(b)] with $h_{0}=\gamma=1 \mathrm{~nm}$ located at the center where $\epsilon=10.0 \mathrm{meV}$ and $\sigma=3.4 \AA$. The inset of Fig. 12(a) shows the far view of GE and the inset of Fig. 12(b) shows the side view of the Gaussian bump. The red colors refer to the highest stresses which are mostly located around the bump region, $r \leqslant 2 \gamma$. For this size of the bump, GE follows the Gaussian bump, i.e., $h_{S}(x, y) \approx h_{1}+h_{G}(x, y)$, where $h_{1}$ is a vertical shift which is about graphite's layer spacing $3.4 \AA$.
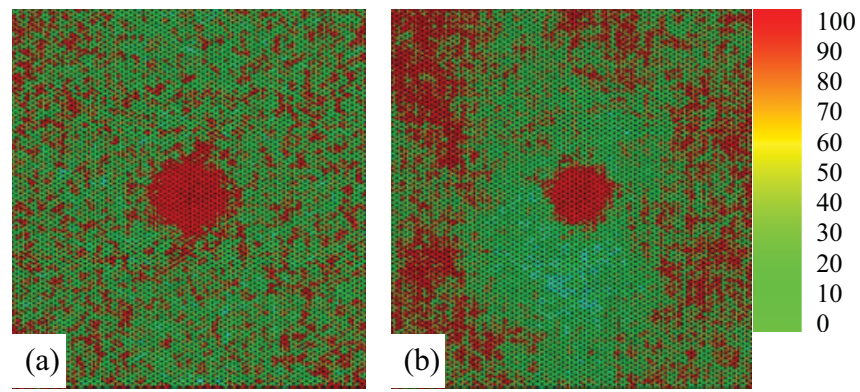

FIG. 14. (Color online) Bond length distribution in graphene on top of a Gaussian bump (a) and a Gaussian depression (b). The longer bond lengths are shown by red colors and the shorter bond lengths are shown by green colors.

Figure 12(c) shows the optimum configuration of GE (found from MD) on top of a depression with $h_{0}=-1 \mathrm{~nm}$ and $\gamma=$ $1 \mathrm{~nm}$. As we see the deformation of the GE over the Gaussian bump is different from the one over the depression (while both have the same variance, heights, and potential parameters, i.e., $\epsilon=10.0 \mathrm{meV}$ and $\sigma=3.4 \AA$ ). This is clear from the curves shown in Fig. 13 which were taken along $x=0$ and $y=0$ [corresponding to the deformations shown in Figs. 12(a) and 12(c)]. The optimum configuration of GE on top of the depression [Figs. 13(c) and 13(d)] is not a Gaussian, i.e., $h_{S}(x, y) \neq h_{1}+h_{G}(x, y)$, because of the stronger repulsive force inside the depression due to the interaction between GE and the substrate. The bond length distribution is shown in Fig. 14.

Figure 15 shows the variation of both $E_{v d W}^{A}$ (MD) [Eq. (3)] and $E_{v d W}^{C}(\mathrm{CM})$ [Eq. (10)] versus the radius, $r$, where $r$ is the upper limit of the integrals in Eq. (10). In Fig. 15(a), we set $\gamma=h_{0}=1 \mathrm{~nm}, \epsilon=10 \mathrm{meV}$, and $\sigma=3.4 \AA$, which are close to the one for the $\mathrm{SiO}_{2}$ substrate. ${ }^{11}$ For the substrate with a bump the energies of the atomistic model (MD) are close to the one obtained from the continuum model (CM). For the depression, our MD results are different from the continuum model results, which is a consequence of the different profiles
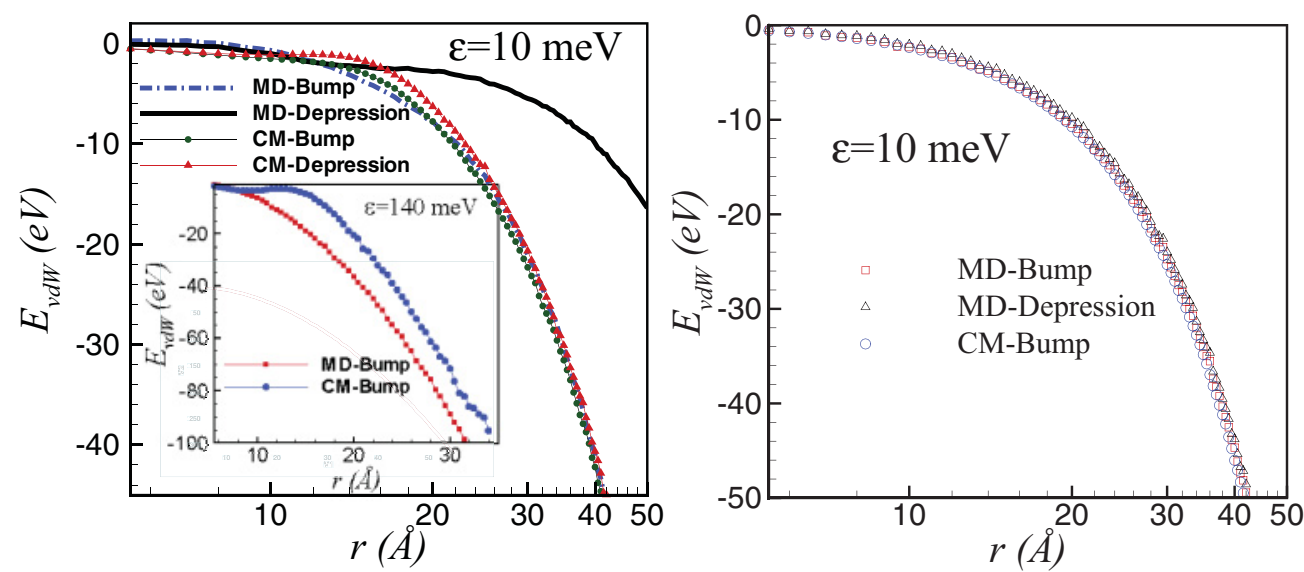

FIG. 15. (Color online) (a) Variation of the van der Waals energy versus the radius (in log scale) measured from the origin (0, 0). Results are presented for both molecular dynamics simulation [MD, Eq. (3)] and continuum model [CM, Eq. (10)]. Here the energy parameter in the LJ potential was set to $10 \mathrm{meV}$ (in the inset we took $140 \mathrm{meV}$ ) and $\sigma=3.4 \AA$. The substrate is a Gaussian bump with $10 \exp \left(-\rho^{2} / 200\right)(\AA)$ deformation. (b) Variation of the vdW energy versus the radius $(r)$ for wider Gaussian bump/depression with $10 \exp \left(-\rho^{2} / 900\right)(\AA)$ deformation with $\epsilon=10 \mathrm{meV}$ and $\sigma=3.4 \AA$. 

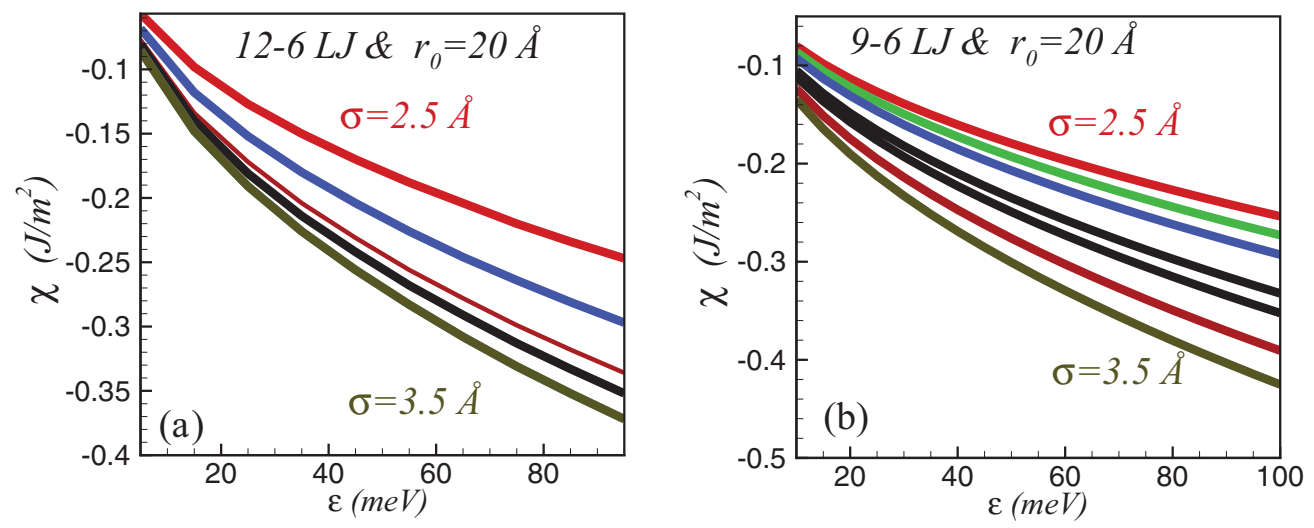

FIG. 16. (Color online) Variation of van der Waals energy per area versus $\epsilon$ from continuum model [Eq. (10)] for graphene on top of a Gaussian bump with $10 \exp \left(-\rho^{2} / 200\right)$ deformation, where $\sigma$ increases from top to bottom with steps of $0.25 \AA$. In (a), (b) we used 12-6 and 9-6 LJ potential parameters, respectively. In both panels $r=r_{0}=2 \mathrm{~nm}$ which is the upper limit of the integrals in Eq. (10) (see the text).

in GE and the substrate (see Fig. 13). Notice that the used profile in CM for graphene on top of a depression is a Gaussian profile (compatible with the substrate profile) while the found optimized profile in the MD simulation as seen from Figs. 13(c) and 13(d) is not a Gaussian. Therefore, there is a significant deviation from the $\mathrm{CM}$ and MD results for depression as shown in Fig. 15(a). In the inset of Fig. 15(a), which is related to graphene on top of a bump, we set the energy parameter to $\epsilon=140 \mathrm{meV}$. When the energy parameter is large, the results of MD and CM deviate, which is related to the strong attraction between the substrate and GE.

Figure 15(b) shows the vdW energy for a Gaussian bump/depression with larger variance, i.e., $\gamma=3 \mathrm{~nm}$ and $h_{0}=1 \mathrm{~nm}, \epsilon=10 \mathrm{meV}$, and $\sigma=3.4 \AA$. There is good agreement between the results of MD simulations and those found from the continuum model (CM). We conclude that for large variance the continuum model provides a vdW contribution to the adhesion energy which is comparable to the MD atomistic results. But for small bump/depression the $\mathrm{CM}$ model is not applicable and the lattice structure of GE should be taken into account.

In Fig. 16 the variations of $\chi=E_{v d W}^{C} / A$ versus $\epsilon$ for various $\sigma(=2.5 \AA, 3.5 \AA)$ are shown. Here, graphene is deposited on top of a Gaussian bump with $\gamma=1 \mathrm{~nm}$ and $h_{0}=$ $1 \mathrm{~nm}$ where $r=r_{0}=2 \mathrm{~nm}$ and the area is calculated using $A=\pi r_{0}^{2}=4 \pi \mathrm{nm}^{2}$. In Fig. 16(a) and Fig. 16(b) we used the 12-6 LJ and the 9-6 LJ potential parameters, respectively. The 9-6 LJ potential gives results that have larger $|\chi|$ for a particular $\epsilon$ (notice that in this paper the 9-6 LJ potential is used only for comparative purposes).

The energy per area, i.e., $\chi$, is in the range of the adhesion energy found for a graphene membrane positioned on top of $\mathrm{SiO}_{2}$ substrate, ${ }^{20}$ i.e., $0.31-0.45 \mathrm{~J} / \mathrm{m}^{2}$. However, note that our results give only the vdW contribution of the adhesion energy which results from the standard $r^{-6}$ dispersion interaction.

\section{Gaussian bump/depression: Supported boundary conditions}

Since the optimum configuration of supported graphene over a Gaussian bump is similar to the one for a spherical bubble, we will not report them here. For supported graphene over a Gaussian depression the optimum configuration is not Gaussian, similarly as for free graphene over a depression (we do not show the optimum configuration here).

\section{SUMMARY}

We carried out several molecular dynamics simulations and studied systematically the optimum configuration of large-scale graphene deposited on top of several differently shaped substrates. The stress distribution in graphene shows that highly stressed atoms are located around the deformed regions of the substrate.

For short wavelength $(\leqslant 2 \mathrm{~nm})$ graphene is suspended across the neighbor peaks of the sinusoidal substrate and thus graphene does not follow the substrate. A graphene sheet on top of a cubic barrier shows an unexpected pyramidal shape. It is found that for large Gaussian bump/depression the van der Waals contribution in the adhesion energy is in agreement with the prediction of the continuum model. The van der Waals adhesion energy per area for a nanoscale Gaussian bump is found to be around $0.1-0.35 \mathrm{~J} / \mathrm{m}^{2}$ depending on the energy parameter of the model.

\section{ACKNOWLEDGMENTS}

We thank L. Covaci and S. Costamagna for valuable comments. We acknowledge M. Zarenia, M. R. Masir and D. Nasr for fruitful discussions. This work was supported by the Flemish Science Foundation (FWO-Vl) and ESF EUROCORE program EuroGRAPHENE: CONGRAN.
${ }^{1}$ K. S. Novoselov, A. K. Geim, S. V. Morozov, D. Jiang, Y. Zhang, S. V. Dubonos, I. V. Gregorieva, and A. A. Firsov, Science 306, 666 (2004).
${ }^{2}$ K. S. Novoselov, A. K. Geim, S. V. Morozov, D. Jiang, M. I. Katsnelson, I. V. Grigorieva, S. V. Dubonos, and A. A. Firsov, Nature (London) 438, 197 (2005). 
${ }^{3}$ D. Nelson, D. Piran, and S. Weinberg, Statistical Mechanics of Membranes and Surfaces (World Scientific, Singapore, 2004).

${ }^{4}$ P. S. Swain and D. Andelman, Langmuir 15, 8902 (1999).

${ }^{5}$ P. S. Swain and D. Andelman, Phys. Rev. E 63, 051911 (2001).

${ }^{6}$ W. Bao, F. Miao, Z. Chen, H. Zhang, W. Jang, C. Dames, and C. Ning Lau, Nature Nanotechnology 4, 562 (2009).

${ }^{7}$ Masa Ishigami, J. H. Chen, W. G. Cullen, M. S. Fuhrer, and E. D. Williams, Nano Lett. 7, 1643 (2007).

${ }^{8}$ J. Sabio, C. Seóanez, S. Fratini, F. Guinea, A. H. Castro Neto, and F. Sols, Phys. Rev. B 77, 195409 (2008).

${ }^{9}$ S. Safran, Statistical Thermodynamics of Surfaces, Interfaces, and Membranes (Addison-Wesley Publishing Company, 1994).

${ }^{10}$ J. F. Dobson, A. White, and A. Rubio, Phys. Rev. Lett. 96, 073201 (2006).

${ }^{11}$ Zhun-Yong Ong and Eric Pop, Phys. Rev. B 81, 155408 (2010).

${ }^{12}$ T. Hertel, R. E. Walkup, and P. Avouris, Phys. Rev. B 58, 13870 (1998).

${ }^{13}$ J. Xiao, S. Dunham, P. Liu, Y. Zhang, C. Kocabas, L. Moh, Y. Huang, K.-C. Hwang, C. Lu, W. Huang, and J. A. Rogers, Nano Lett. 9, 4311 (2009).

${ }^{14}$ M. Neek-Amal, N. Abedpour, S. N. Rasuli, A. Naji, and M. R. Ejtehadi, Phys. Rev. E 82, 051605 (2010).

${ }^{15}$ M. Neek-Amal and F. M. Peeters, Phys. Rev. B 81, 235421 (2010).

${ }^{16}$ A. I. Zhbanov, E. G. Pogorelov, and Y-C. Chang, ACS Nano 4, 5937 (2010).

${ }^{17}$ N. Marom, J. Bernstein, J. Garel, A. Tkatchenko, E. Joselevich, L. Kronik, and O. Hod, Phys. Rev. Lett. 105, 046801 (2010).

${ }^{18}$ J. Zhao, A. Buldum, J. Han, and J. Ping Lu, Nanotechnology 13, 195 (2002).
${ }^{19}$ C. Thierfelder, M. Witte, S. Blankenburg, E. Rauls, and W. G. Schmidt, Surf. Sci. 605, 746 (2011).

${ }^{20}$ S. P. Koenig, N. G. Boddeti, M. L. Dunn, and J. Scott Bunch, Nature Nanotechnology 6, 543 (2011).

${ }^{21}$ S. Viola Kusminskiy, D. K Campbell, A. H. Castro Neto, and F. Guinea, Phys. Rev. B 83, 165405 (2011).

${ }^{22}$ D. W. Brenner, O. A. Shenderova, J. A. Harrison, S. J. Stuart, B. Ni, and S. B. Sinnot, J. Phys.: Condens. Matter 14, 783 (2002).

${ }^{23}$ Zhixing Lu and Martin L. Dunna, J. Appl. Phys. 107, 044301 (2010).

${ }^{24}$ M. Neek-Amal, R. Asgari, and M. R. Rahimi Tabar, Nanotechnology 20, 135602 (2009).

${ }^{25}$ S. Erkoc, Annual Reviews of Computational Physics IX, edited by D. Stauffer (World Scientific, Singapore, 2001).

${ }^{26}$ N. Chandra, S. Namilae, and C. Shet, Phys. Rev. B 69, 094101 (2004).

${ }^{27}$ Q. X. Pei, Y. W. Zhang, and V. B. Shenoy, Carbon 48, 898 (2010).

${ }^{28}$ J-M. Y. Carrillo, E. Raphael, and A. V. Dobrynin, Langmuir 26, 12973 (2010).

${ }^{29}$ P. Lauffer, K. V. Emtsev, R. Graupner, Th. Seyller, L. Ley, S. A. Reshanov, and H. B. Weber, Phys. Rev. B 77, 155426 (2008).

${ }^{30}$ T. Georgiou1, L. Britnell, P. Blake, R. V. Gorbachev, A. Gholinia, A. K. Geim, C. Casiraghi, and K. S. Novoselov, Appl. Phys. Lett. 99, 093103 (2011).

${ }^{31}$ E. Stolyarova, D. Stolyarov, K. Bolotin, S. Ryu, L. Liu, K. T. Rim, M. Klima, M. Hybertsen, I. Pogorelsky, I. Pavlishin, K. Kusche, J. Hone, P. Kim, H. L. Stormer, V. Yakimenko, and G. Flynn, Nano Lett. 9, 332 (2009).

${ }^{32}$ M. Neek-Amal and F. M. Peeters, J. Phys.: Condens. Matter 23, 045002 (2011). 